\title{
L'héritage colonial et les langues en Afrique francophone
}

Colonial legacy and language in French-speaking west Africa

La herencia colonial y las lenguas en el África francófona

\section{A. Racine Senghor}

\section{OpenEdition}

\section{Journals}

Édition électronique

URL : http://journals.openedition.org/ries/1708

DOI : $10.4000 /$ ries. 1708

ISSN : 2261-4265

Éditeur

Centre international d'études pédagogiques

Édition imprimée

Date de publication : 1 septembre 2003

Pagination : 77-85

ISBN : 978-2-85420-557-1

ISSN : $1254-4590$

Référence électronique

A. Racine Senghor, "L'héritage colonial et les langues en Afrique francophone », Revue internationale d'éducation de Sèvres [En ligne], 33 I septembre 2003, mis en ligne le 06 juin 2012, consulté le 19 avril 2019. URL : http://journals.openedition.org/ries/1708 ; DOI : 10.4000/ries.1708 


\section{L’héritage colonial et les langues en Afrique francophone}

\section{A. Racine Senghor}

La question de l'enseignement des langues vivantes en Afrique francophone sera examinée à partir de l'observation de plusieurs pays: le Bénin, le Burkina, le Mali, Madagascar, le Sénégal et, dans une moindre mesure, la Guinée et le Niger. Suffisamment représentatifs par l'originalité de leur démarche, ces pays nous permettront de donner une idée de ce qui se fait, dans cette partie du monde, en matière d'enseignement des langues vivantes. Les informations que nous livrons ici proviennent d'une enquête effectuée dans certains pays du réseau ARCHES, qui est un projet pour l'harmonisation des enseignements secondaires dans environ seize pays d'Afrique francophone et de l'Océan Indien. Nous partirons d'un rappel historique, avant de voir les perspectives politiques puis les finalités de cet enseignement.

\section{De L'ÉCOLE COLONIALE AUX ÉCOLES NATIONALES}

A Saint-Louis du Sénégal débarqua, en 1816, l’instituteur Jean Dard qui ouvrit la première école en Afrique. Il commença par apprendre la langue locale et entreprit de former ses élèves avec cette langue, anticipant ainsi sur un débat qui est aujourd'hui d'actualité: l'introduction des langues nationales à l'école. Mais l'expérience de ce maître plein d'audace ne dura qu'une année...

Dans l'espace francophone, l'école africaine, est héritière de l'école française. Ainsi d'ailleurs l'appelle-t-on encore de nos jours, par opposition à l'école coranique ou arabe ou à cette autre école traditionnelle que représenterait, par exemple, la réclusion initiatique au moment de la circoncision. Si elle avait indubitablement ses spécificités du fait des objectifs particuliers qui lui étaient assignés dans le cadre de la colonisation, elle était souvent, notamment au collège et au lycée, une simple copie des écoles de l'Hexagone.

Les langues vivantes enseignées étaient les mêmes que celles qu'étudiaient les jeunes fréquentant des établissements scolaires en France, avec les mêmes programmes, les mêmes ouvrages et les mêmes certifications. C'est à l'académie de Bordeaux qu'étaient rattachés les pays d'Afrique.

En série classique, les élèves apprenaient le latin, le grec, l'arabe classique et des langues vivantes, comme leurs camarades de la série moderne: l'anglais, l'espagnol, l'allemand, le russe, etc. L'anglais était généralement la première langue vivante pour tous à partir de la sixième (rarement, c'était l'alle- 
mand); et à partir de la quatrième, une deuxième langue vivante devait être choisie sauf pour les élèves qui suivaient l'enseignement court dans un type particulier de collège n'offrant, aux élèves souvent très âgés, aucune possibilité de poursuivre leurs études après le brevet du premier cycle.

Puis vinrent les indépendances, en 1960 pour la quasi totalité des pays, accompagnées souvent de la volonté légitime de bâtir une école correspondant à l'idée que l'on se faisait du devenir de l'État et de la nation à bâtir.

\section{LeS PERSPeCtIVES POLITIQUES}

Dans les écoles considérées, l'enseignement, bien naturellement, se fait en français, langue seconde, mais aussi langue officielle de l'administration et des affaires. Ce statut, il n'est pas près de le perdre, même si se pose de manière de plus en plus nette la nécessité de faire entrer les langues nationales à l'école.

Il est important de noter la place particulière de l'arabe dans certains pays où l'islam constitue la religion majoritaire. Langue d'enseignement dans les medersa du Mali et du Sénégal, l'arabe est aussi, dans ce dernier pays, langue d'enseignement au lycée public franco-arabe qui reçoit des élèves issus des écoles primaires publiques ou privées francophones ou franco-arabes.

\section{LANGUES ANCIENNES}

A côté, donc, du français sont enseignées d'autres langues, vivantes ou anciennes. La série classique, celle des langues anciennes (le latin et le grec) existe encore aujourd'hui dans certains pays, mais en nombre très réduit. Jadis lieu d'accueil des meilleurs élèves, "les forts en thème", elle est aujourd'hui désaffectée. Au Sénégal, elle se retrouve dans une dizaine d'établissements publics et surtout dans les collèges privés confessionnels et les séminaires. Il en est de même pour le Bénin. Dans les autres pays, seuls les établissements à caractère religieux dispensent cet enseignement. L'arabe classique aussi a disparu des écoles, laissant la place à l'arabe langue vivante enseignée comme deuxième langue.

Le rétrécissement de la série classique trouve son explication dans plusieurs facteurs parmi lesquels: le caractère ancien, donc inutilisable de ces langues (mortes !) au moment où le besoin de communication prend une importance particulière dans le monde moderne; leur hermétisme qui rend leur apprentissage ardu; l'important volume horaire, à l'école comme à la maison, que les élèves consacrent à ces matières. Bref, on considère que ce sont des disciplines qui demandent aux enfants une surcharge de travail, sans que l'on ressente vraiment leur utilité. S'y ajoute que, face au manque de professeurs dans ces pays, les professeurs de lettres classiques sont vite appelés à se limiter à l'enseignement du français, matière obligatoire à tous les niveaux. 
L'ancien président du Sénégal, Léopold Sédar Senghor, agrégé de grammaire et pur produit des humanités classiques, accordait, du temps de sa magistrature, une importance toute particulière à l'enseignement des langues anciennes pour la formation de l'esprit des jeunes à la rigueur, à la discipline et à la méthode, toutes choses qui contribuent à la formation de l'esprit scientifique. Senghor a même tenté de sauver le latin en favorisant une méthode «vivante» d'enseignement de cette langue, en liaison avec le Centre de linguistique appliquée de Dakar (CLAD). Mais cette méthode, Africani Latine Discunt, fit long feu et disparut dès que son initiateur quitta le pouvoir.

Il n'est donc pas osé de dire que les langues anciennes sont vraiment en voie de disparition sans qu'aucune mesure ne se dessine pour leur survie pour le moment.

\section{LANGUES VIVANTES}

L'anglais occupe la première place parmi les langues vivantes. Il est enseigné partout comme première langue (LV1) de la classe de sixième à la classe de terminale. Puis viennent les deuxièmes langues (LV2) dont l'enseignement se fait à partir de la classe de quatrième, au choix de l'élève, en fonction des offres de langues de l'établissement. Dans l'ordre de leur importance par le nombre des élèves concernés, nous avons l'espagnol, l'allemand, l'arabe, le portugais, le russe et, à niveau sensiblement égal, l'italien et le chinois. Ces langues étaient déjà présentes à l'époque coloniale, sauf le chinois que l'on retrouve au Mali uniquement.

Lorsqu'on y regarde de près, l'on se rend compte que ces langues correspondent aux grandes langues de communication internationale. C'est là sans doute la raison principale de leur choix ou de leur maintien dans les différents systèmes éducatifs africains. Il importe en effet d'ouvrir les jeunes au monde et de favoriser la participation du pays et de ses cadres, en particulier, à la vie globale. L'apprentissage d'une langue constitue aussi la voie royale pour se familiariser avec la culture qu'elle véhicule et s'enrichir de ce qu'apporte nécessairement cette culture différente. Une autre raison serait liée à des choix politiques: c'est le cas du russe pendant un moment en Guinée et celui du chinois au Mali. À une période de leur histoire, ces deux pays ont entretenu avec ces puissances des relations privilégiées qui ont, par conséquent, permis "l'élection» de leurs langues dans les écoles. L'arabe, langue du Saint Coran, occupe une importante place dans les pays fortement islamisés comme le Mali, le Niger, le Sénégal et le Tchad. Il faut dire aussi que des raisons stratégiques militent en faveur de cette langue: les pays arabes, particulièrement riches, sont sollicités par les pays en développement et la présence de leur langue dans les écoles peut constituer un argument fort pour drainer les pétrodollars... Par ailleurs, sans que l'on ait de mesures précises sur la question, les pays d'accueil 
des fortes colonies d'immigrés africains devraient voir se développer leurs langues en Afrique.

Au niveau de l'école élémentaire, c'est le français qui est le plus largement enseigné et qui est langue d'enseignement. On y trouve aussi l'arabe, dans certains pays, avec des crédits horaires variant de deux heures au Sénégal à six ou huit dans les medersa, écoles franco-arabes, du Mali, du Niger... Les langues nationales, de plus en plus, sont introduites dans les systèmes éducatifs, à l'élémentaire en particulier, comme discipline ou comme langue d'enseignement.

Sauf dans les facultés et autres instituts universitaires, nous n'avons pas connaissance d'enseignement de langues africaines dans les pays autres que leur pays d'origine. Il existe cependant des langues transversales, parlées dans plusieurs parties du continent, qui sont bien entendu enseignées à une vaste échelle, avec les variantes qui les caractérisent. Les deux exemples qui viennent à l'esprit sont le pulaar (appelé aussi peul ou fulfuldé) et le swahili.

\section{LES FINALITÉS DE L'ENSEIGNEMENT DES LANGUES}

\section{Les objectifs}

Les objectifs généraux de l'enseignement des langues, comme indiqué plus haut, portent sur le besoin, pour des raisons diverses, de doter le citoyen de moyens lui permettant de se bien mouvoir dans le monde par la maîtrise au moins, en plus du français, d'une ou de deux langues de communication internationale.

Les objectifs pédagogiques sont pour l'essentiel: lire/écouter correctement; comprendre ce qu'on a lu ou entendu; écrire/parler correctement. En somme, il s'agit d'arriver à communiquer, à l'écrit comme à l'oral, selon les situations. Le crédit horaire, pour atteindre ces objectifs au niveau du collège, est de quatre à cinq heures/semaine pour la LV1, dont quatre heures par élève (la classe devant être dédoublée pour permettre un travail rapproché comme pour les travaux dirigés ou les cours d'expression orale). Au niveau du lycée, en fonction des séries, deux à quatre heures sont consacrées à cet enseignement.

\section{Les approches méthodologiques}

La méthode traditionnelle cède progressivement la place à la méthode structurale mais surtout à la méthode communicative. La première méthode consiste à mettre l'accent sur le texte écrit, sur la lecture et sur la traduction dans un sens ou dans un autre(version, thème). Elle a fait beaucoup de victimes. Sortis du lycée, de nombreux élèves sont incapables de formuler la moindre phrase correcte dans une langue qu'ils ont mis cinq ou sept ans à étudier. Puis 
vint l'approche structurale, qui marque quelque évolution, mais qui ne réalise pas l'autonomie de l'élève, même si elle lui permet d'acquérir des automatismes. En fait, elle confère seulement des automatismes pour des situations données, en dehors desquelles l'élève éprouve des difficultés à communiquer. De plus en plus souvent, maintenant, il est fait usage de la méthode communicative, plus fonctionnelle et plus à même de conduire à la satisfaction des objectifs pédagogiques définis plus haut: comprendre et répondre à l'écrit et à l'oral.

Cette dernière approche gagne donc petit à petit du terrain, mais son plein épanouissement se heurte à des problèmes cruciaux tels que le déficit de formation des enseignants, l'indigence matérielle (infrastructures, équipement, manuels) et les effectifs pléthoriques qui défient les imaginations les plus aventureuses (parfois quatre-vingts, cent, cent vingt... deux cents élèves par classe dans certains pays). Dans de telles conditions, il est difficile, vraiment, d'appliquer une méthode communicative ou même quelque autre méthode que ce soit. Des formules existent et sont expérimentées pour l'enseignement dans les grands groupes. Mais dans la plupart des cas, il s'agit de plus que de grands groupes!

Les cassettes audio et vidéo sont largement utilisées, mais très rarement l'outil informatique qui constitue presque partout un véritable luxe. L'usage de ces cassettes n'est pas non plus partout répandu; il dépend d'ailleurs davantage de l'engagement personnel du professeur et de ses moyens propres que de la mise à disposition par l'administration scolaire des outils nécessaires.

Des écoles bilingues sont ouvertes dans quelques pays. Nous avons évoqué les établissements (privés surtout) franco-arabes, les medersa, où les deux langues sont à la fois langues d'enseignement et discipline. Le bilinguisme anglais-français est aussi en train de se frayer son chemin. Dans les grandes villes, comme Dakar, existent des écoles de ce type au niveau du secondaire qui sont le résultat d'initiatives privées. Ces écoles sont très prisées, notamment par les couches aisées de la société, seules capables d'honorer les frais d'écolage hors de portée des faibles bourses.

Les centres culturels des ambassades des pays du nord dispensent aussi des cours de langue (de leur langue) particulièrement appréciés des jeunes et des moins jeunes. Les plus dynamiques concernent l'anglais et l'allemand, mais aussi le français. Notons que les structures culturelles françaises en Afrique dispensent également des cours de langues nationales.

\section{L’ÉVALUATION}

Les langues vivantes, objet d'enseignement figurant normalement dans les programmes, font l'objet d'une évaluation. Elles sont affectées d'un coefficient qui varie de quatre à deux selon qu'il s'agisse d'une langue vivante $1 \mathrm{ou}$ d'une langue vivante 2 , selon qu'on se trouve en série littéraire ou en série 
scientifique. Au collège, d'une manière générale, le coefficient de la LV1 est de quatre quand celui de la LV2 est de trois. Au lycée, il est de trois en série littéraire (et peut atteindre quatre en série littéraire 2), et de deux en série scientifique.

Ces coefficients s'appliquent en classe pour l'évaluation formative, mais aussi à l'examen au moment de l'évaluation certificative. Ils dénotent la place importante des langues dans le système éducatif, pour la réussite ou l'échec de l'élève, surtout au niveau du collège. A titre comparatif, les coefficients tournent autour de quatre pour les mathématiques, deux pour l'orthographe, deux pour la composition française...

A l'examen, il est généralement prévu et administré une épreuve écrite et une épreuve orale de langue vivante, aussi bien au baccalauréat qu'au brevet de la classe de troisième (le BEPC ) dont l'appellation varie selon les pays. Les coefficients sont plus élevés à l'écrit qu'à l'oral.

Dans certains pays, l'épreuve de langue en série scientifique se réduit à l'épreuve orale, tandis que dans d'autres la tendance est à la suppression pure et simple de l'oral, non parce que sa nécessité est remise en cause, mais tout simplement parce que les conditions de son déroulement lui enlèvent souvent toute crédibilité.

À l'école élémentaire, le seul exemple de langue vivante étrangère enseignée est l'arabe. Dans les pays où il est langue d'enseignement, les évaluations se font aussi dans cette langue et en français. Il y est aussi évalué en tant que discipline. Par contre, dans des pays comme le Sénégal, l'arabe est enseigné au primaire (public) en tant que langue vivante mais aussi un peu comme matière facultative qui, par conséquent, n'intervient nullement dans l'évaluation certificative des élèves.

\section{LES DÉBATS ACTUELS}

\section{La question des langues nationales}

Les débats actuels ne concernent ni le choix ni la place des langues vivantes étrangères dans le système éducatif. Sauf que dans certains pays, compte tenu des difficultés à pourvoir les établissements en personnel, certaines langues ont été supprimées. Ainsi en est-il du Niger où la seule langue vivante 2 enseignée est l'arabe, après la suppression de l'espagnol. L'anglais y reste langue vivante 2.

L'introduction des langues nationales dans les écoles, leur place dans le système, les langues à choisir si choix il devrait y avoir, voilà quelques unes des questions qui, aujourd'hui, constituent le principal débat. Celui-ci relève d'abord de considérations politiques parce qu'on invoque la nécessité de valoriser les langues nationales. Celles-ci, pense-t-on, devraient être élevées à la même dignité que les 
langues étrangères, langues d'emprunt, fussent-elles langues officielles. La voie royale, pour ce faire, est que les enfants puissent y être formés en même temps que s'organisent des cours d'alphabétisation dans ces langues en direction des adultes et de tous ceux qui n'ont pu accéder à l'école.

Mais le débat trouve aussi sa justification dans la recherche de l'efficacité pédagogique. On part du principe selon lequel il y a plus à gagner si les premières acquisitions de l'enfant se font dans sa propre langue. De nombreuses expériences sont en cours dans la quasi totalité des pays pour l'introduction de ces langues. Mais la question n'est pas simple.

D'abord, les langues peuvent être très nombreuses et se pose alors la difficile question des choix à opérer ou simplement de la maîtrise nécessaire à leur enseignement. Il est bon de rappeler ici la position de Léopold Sédar Senghor qui fut parmi les premiers à défendre l'enseignement des langues nationales. Il soutenait que l'un des préalables était que soient suffisamment étudiées ces langues du point de vue de leur description scientifique. Et que les maîtres devaient être formés. D'autre part se pose le problème pratique de la ou des langue(s) à enseigner, en un lieu donné, sans réveiller le démon des susceptibilités, quand on sait que de plus en plus, avec les mouvements des populations, plusieurs langues peuvent se retrouver dans le même espace.

Ensuite, selon quel processus introduire ces langues? Selon quel rapport au français? Comment organiser la cohabitation entre le français et les langues nationales? Avec quels moyens didactiques et pédagogiques?

Le Mali expérimente depuis plusieurs années une pédagogie convergente langues nationales/français. Elle reste à être évaluée et étendue à l'ensemble du pays. Ses résultats aideraient sans doute les autres pays à mieux s'y prendre en les confrontant bien sûr à leurs propres expériences.

Madagascar et la Mauritanie ont également tenté l'un la "malgachisation», l'autre l'arabisation pour répondre à des impératifs politiques les détournant du français. Ils sont revenus en partie à celui-ci, du fait que leur option n'a pas toujours donné les résultats escomptés. D'où la nécessité, encore une fois, de prendre toutes les dispositions préparatoires qui aident à s'élancer du bon pied.

\section{Le cas spécifique de Madagascar}

Comme la Mauritanie donc, Madagascar constitue un cas particulier. Pas seulement parce que le malagasy (lire malgache), qui est la seule langue nationale de cette vaste île, avait supplanté le français avant que l'on reconsidère ce choix ! En effet depuis la rentrée des classes 2002-2003, l'État a décidé (et appliqué) l'introduction à titre expérimental de l'enseignement/apprentissage de la langue de Shakespeare à l'école élémentaire, en clase de huitième, c'est-à-dire au CM1. 
Mais les débats restent ouverts au sein de la société de faire de l'anglais, à long terme, la troisième langue d'enseignement à côté du malagasy et du français. On ne perd pas de vue, bien entendu, que la réalisation d'un tel projet nécessite des moyens colossaux.

$$
\text { ** }
$$

En conclusion, nous pouvons retenir que l'école africaine d'aujourd'hui n'a pas renié l'héritage que lui a laissé l'école coloniale pour ce qui est de l'enseignement des langues vivantes. Celles-ci continuent à être présentes dans les systèmes éducatifs, même si des réalités particulières amènent souvent à les reconsidérer et à réévaluer leur nombre. L'Afrique est en effet un continent où sévit le sous-développement et où l'école, bien que son importance n'échappe à personne, se construit dans la difficulté. Les langues vivantes sont enseignées pour ce qu'elles représentent dans le monde, c'est-à-dire pour l'ouverture qu'elles offrent en tant que grandes langues de communication internationale. Mais aujourd'hui se pose surtout la question de l'introduction des langues nationales dans le système éducatif. C'est une question sérieuse qui pourrait déterminer le devenir de ces États qui se cherchent encore et dont le salut passe nécessairement par la formulation la plus correcte possible du rôle de l'école. Celle-ci, pour reprendre le mot de Senghor, devrait se fonder sur une double postulation : l'enracinement et l'ouverture. «S'enraciner dans les valeurs de sa propre civilisation et s'ouvrir aux apports fécondants de l'extérieur.» Les langues vivantes étrangères et les langues nationales conduisent à l'une et l'autre postulations. 


\section{Organisation de l'enseignement des langues au Sénégal}

\section{Enseignement primaire}

Enseignement fondamental en Français, classes expérimentales en langues nationales avec introduction progressive du Français à partir de la $3^{\mathrm{e}}$ année. L'arabe est enseigné à l'élémentaire pendant 2 ans par des maitres formés et titulaires

\section{Enseignement secondaire}

Enseignement des disciplines fondamentales en Français. Langues enseignées : LV1 : Anglais 208139 élèves ; LV2 : espagnol 46113 arabe 22099 ; portugais 8697 ; allemand 7412 ; russe 1354 ; italien 215 . La LV1 est obligatoire pour tous de la $6^{\mathrm{e}}$ à la terminale. Choix obligatoire d'une LV2 à partir de la $4 \mathrm{e}$.

\section{Enseignement public/privé}

Pas de variation.

\section{Langues anciennes}

Dans une dizaine de collèges et lycées publics et la quasi totalité des écoles privées catholiques, mais aussi les séminaires enseignent les langues anciennes (le latin plus que le grec).

\section{Langues régionales}

Non.

\section{Langues de l'immigration}

Non

\section{Expérimentations}

Il existe des écoles privées bilingues français/anglais, ainsi que des instituts pour l'apprentissage de l'anglais (surtout) et de l'allemand.

\section{Place des langues dans les certifications}

BFEM (équivalent du BEPC français): anglais, LV1: coefficient 3 à l'écrit et à l'oral; LV2: coefficient 2 à l'écrit et à l'oral (option). Baccalauréat: en série L (écrit et oral), coefficient 4, en série $\mathrm{S}$ (oral), coefficient 2.

\section{Grilles d'évaluation internationales}

Non, sauf pour les Instituts de langue. 
